\title{
Identification and Reporting of Gender Identity in HIV Surveillance Data in the Deep South
}

\author{
Susan Reif ${ }^{1}$ [ Elena Wilson ${ }^{1} \cdot$ Haley Cooper $^{1} \cdot$ Genevieve Hunter $^{1} \cdot$ Carolyn McAllaster $^{2}$ \\ Accepted: 27 December 2021 / Published online: 20 January 2022 \\ (c) The Author(s), under exclusive licence to Springer Science+Business Media, LLC, part of Springer Nature 2022
}

\begin{abstract}
Introduction Although studies have identified high prevalence of HIV among individuals who are transgender, HIV surveillance data regarding gender identity is incomplete, resulting in uncertainty regarding the gender identity, including transgender and other diverse gender identities, of individuals diagnosed with HIV. This information is critical to planning strategies for HIV prevention and care.

Methods From August 2018 to March 2019, interviews were conducted with HIV surveillance leadership (including Surveillance Directors, Epidemiologists, and HIV/STI Prevention Staff) at offices of epidemiology from eight US Deep South states regarding their practices related to gender identity documentation in HIV surveillance data and the barriers encountered in these documentation processes as well as their recommendations for improving gender identity data collection.

Results Interview findings indicated significant barriers to collection of accurate gender identity information in HIV surveillance data including lack of standardized data systems for collecting gender identity; difficulty obtaining gender identity information from HIV testing sites, laboratories, and medical databases; and need for enhanced cultural sensitivity and gender identity knowledge at all levels of the data collection process. Recommendations from the state HIV surveillance staff, leaders, and epidemiologists are included in the findings.

Conclusions and Policy Implications Effective, well-coordinated strategies are needed to improve gender identity information in HIV surveillance reporting. Recommendations include standardizing and enhancing data collection strategies, providing cultural sensitivity training at all levels of HIV testing/reporting, and developing formal guidance and providing technical assistance that targets and educates laboratories and medical organizations to implement systems of data collection that routinely and safely capture gender identity data.
\end{abstract}

Keywords Gender identity $\cdot$ HIV $\cdot$ AIDS surveillance $\cdot$ Epidemiology $\cdot$ Transgender

\section{Introduction}

The Southern United States (US) has been disproportionately affected by HIV, with the region consistently having the highest HIV diagnosis rates of any US region (Center for Disease Control and Prevention, 2019a, b). A subset of the

Susan Reif

susan.reif@duke.edu

Carolyn McAllaster

mcallaster@law.duke.edu

1 Center for Health Policy and Inequalities Research, Duke University, Durham, USA

2 Colin W. Brown Clinical Professor Emerita of Law, Duke University, Durham, USA
Southern states, often referred to as the Deep South region (defined herein as Alabama, Florida, Georgia, Louisiana, Mississippi, North Carolina, South Carolina, Tennessee, and Texas) (Centers for Disease Control and Prevention, 2019a), share similarities in terms of high HIV diagnosis and death rates and lower attainment on social determinants of health such as economic stability and education access and quality, as well as social and community factors including structural racism and a conservative philosophy that may perpetuate HIV-related stigma and discrimination (Center for Disease Control and Prevention, 2019a; Reif et al., 2016). The Deep South has experienced the highest HIV diagnosis rates and number of people diagnosed with HIV over the last decade along with the highest HIV death rates of any US region (Center for Disease Control and Prevention, 2019b; Reif \& Wilson, 2017; Reif et al., 2014). 
In the US, transgender communities have been particularly impacted by HIV, having significantly higher HIV diagnosis rates than the general population. A systematic review of studies published from 2006-2017 found that $14 \%$ of transgender women, $3 \%$ of transgender men, and 9\% of transgender people overall are living with HIV, compared to $0.5 \%$ of all US adults (Becasen et al., 2018; Center for Disease Control and Prevention, 2019c). Transgender women of color are even further affected, with an estimated $44 \%$ of Black/African American transgender women and $26 \%$ of Hispanic/Latinx transgender women living with HIV, compared to $6.7 \%$ of White transgender women (Becasen et al., 2018). A CDC report regarding HIV prevalence, risk, prevention, and testing among 1608 transgender women living in seven US cities from 2019 to 2020 found that transgender women of color had the highest percentages of new HIV diagnoses in these seven cities (Center for Disease Control and Prevention, 2021a).

Transgender communities face unique challenges that may increase vulnerability to HIV including stigma and discrimination in housing, health care, employment, and law enforcement settings as well as difficulty accessing basic necessities such as affordable housing and affirming medical care (Becasen et al., 2018; Centers for Disease Control and Prevention, 2019c; National Center for Transgender Equality, 2019). A study using HIV surveillance data found that approximately half of transgender people (43\% of transgender women; $54 \%$ of transgender men) who received an HIV diagnosis in the US from 2009 to 2014 lived in the South (Clark et al., 2017). For transgender people living in the South, transphobia, stigma, and violence are often magnified due to the conservative culture and history of systemic racism (Association APHA, 2020; Pichon et al., 2020; Sausa et al., 2009).

To inform policies and interventions that address the critical need for HIV prevention and treatment and social services for transgender and non-binary individuals, it is imperative to accurately collect gender identity for people diagnosed with HIV (Sausa et al., 2009). The Department of Health and Human Services (HHS) included in their Healthy People 2030 objectives to increase the number of states that are collecting gender and sexual identity information in state surveys and data collection and to increase the number of national surveys that collect data on transgender populations, which would include HIV surveillance data, in an attempt to address the lack of comprehensive data for this population (Healthy People 2030, n.d.). In an effort to improve accuracy of gender identity data, the CDC suggested that states adopt a two-step data collection method that involves asking for both sex assigned at birth and current gender identity (Centers for Disease Control and Prevention, 2019c). The CDC's HIV Incidence and Case Surveillance Branch (HICSB) revised HIV surveillance and data collection methods for transgender people in 2011 to align with the aforedescribed two-step model, adding "current gender identity" to the Adult HIV Confidential Case Report Form (ACRF), the form used to compile HIV testing data and to report HIV status and demographic information of individuals who are tested for HIV (Balagi, 2019). However, the only response option for non-binary individuals included in the ACRF was "additional gender identity," and gender identity was not a mandatory response field in data collection.

In 2018, the CDC's HIV Surveillance Report included additional gender identity categories including "transgender MF," "transgender FM," and "additional gender identity" for the first time (Center for Disease Control and Prevention, 2020a, 2018). In the 2018 and 2019 Surveillance Reports, a caveat was included regarding the small number of new diagnoses classified as transgender or having an additional gender identity and the need for supplemental data to more accurately characterize HIV among transgender individuals (Center for Disease Control and Prevention, 2018, 2020, 2021b).

Although availability of gender identity information in HIV surveillance data has increased in recent years, this information is reportedly incomplete and therefore not adequately representative of gender identity among individuals diagnosed with HIV. This report focuses on gender identity information collected in HIV surveillance data in Deep South states, which have been disproportionately impacted by HIV. Two-thirds of the Deep South States reported information on gender identity in their state surveillance data (2018-2019) and the percentage of individuals who identified as transgender was below $2.6 \%$ in all of these states (Alabama Division of STD Prevention and Control HIV Surveillance Branch, 2020; Department of Health, n.d.; DHEC, 2020; Florida Department of Health, 2020; Health, 2019; HIV/STD/Hepatitis Surveillance Unit NC Department of Health and Human Services, 2020; State of Louisiana Department of Health, 2020; TB/HIV/ STD Epidemiology and Surveillance Branch, 2021; Tennessee Department of Health, 2021). This is consistent with the overall percentage reported in HIV surveillance data in the US, $2 \%$ (2018). Identifying practices and related gaps in collection of gender identity information in HIV surveillance data can assist in developing strategies to obtain more complete and accurate gender identity information among people living with HIV (PLWH). Accurate epidemiologic data is essential to inform interventions for transgender individuals living with or at greater risk for HIV in the Deep South and to better tailor allocation of limited resources for transgender people.

\section{Methods}

From August 2018 to March 2019, phone interviews and email follow-ups were conducted with HIV surveillance leadership from eight of the nine Deep South states, 
including four Directors of HIV/STI Surveillance, six State Epidemiologists, six HIV/STI Prevention Staff/Managers, and one HIV/STD Research and Evaluation Director at the state offices of epidemiology. Correspondents were interviewed regarding the practices of gender identity documentation in HIV surveillance data in their state. Leadership from Alabama's surveillance branch could not be reached for interview; a transition in leadership was reported. The purpose of this project was to gather information for a foundation-funded advocacy project, rather than research in nature, thus IRB approval was not sought nor were participants compensated for their time. However, the participants were assured that data would be protected and that participant perspectives would be aggregated to avoid revealing perceptions from specific states/respondents.

Interviews covered the following questions:

- How does the state collect data on individuals diagnosed with HIV?

- What questions do states ask about gender identity for surveillance reporting?

- In what ways do they obtain and verify gender identity information?

- What are the successes and barriers for collecting gender identity data for PLWH?

In-depth notes were taken during interviews, and the information was analyzed to identify themes across interview participants and states.

\section{Results}

\section{Deep South State Collection of Gender Identity Data}

States use the CDC Enhanced HIV/AIDS Reporting System (eHARS), which is a "browser-based, CDC-developed application that assists health departments with reporting, data management, analysis, and transfer of data to the CDC" (Centers for Disease Control and Prevention, 2009; Cohen et al., 2014). Data collected during the HIV testing process or at a later date is used to populate the eHARS data system including sex assigned at birth and current gender identity. Interview participants uniformly noted that collecting accurate data on gender identity for individuals receiving a positive HIV test was challenging. One of the challenges to collecting gender identity information was a lack of standardized data collection tools and computer database platforms within and across states. The states are currently using a variety of ways to obtain data to populate gender identity fields in eHARS including utilizing the CDC-recommended Adult HIV Confidential Case Report Form (ACRF) (Centers for Disease Control and Prevention, 2018). However, interview participants reported significant barriers to accurate reporting of gender identity data including missing gender identity information on ACRFs and delays in submission of the forms to state health departments. One interview participant reported that many of the medical providers in their state will not complete the ACRF, rather these forms are primarily completed by Disease Intervention Specialists (DIS), professionals who are charged with providing education and partner notification services for individuals testing positive for HIV. In another state, field epidemiologists routinely complete the ACRF by contacting providers and/or accessing medical records and reported barriers to reaching providers and incomplete medical record information.

An additional frequently cited barrier to gender identity data collection was a lack of understanding of gender identity categories included on reporting forms among those collecting the data. One example was provided by an interview participant who said, "One factor I recently became aware of is that some reporters may not be sure at what point they should check a person off as transgender on the case report form. Our staff have encountered office staff who may think, for example, that a person needs to have had gender reassignment surgery, which could limit who they identify as transgender." In a related concern, there is a lack of consistency as to whether the individual receiving the positive test is reporting their own gender identity or whether their gender identity is being surmised by someone else, such as the individual providing the test, a DIS worker, or health care provider. Finally, many reporting systems were identified as not having a breadth of options that fall under the transgender spectrum, such as genderqueer or non-binary, which also leads to undercounting of individuals with diverse gender identities.

\section{Supplementing Missing Gender Identity Data}

To address the lack of consistently reported gender identity information, interview participants reported a variety of methods to supplement these data. These methods differed both across states and within states. For example, in one state, the data on a positive HIV test may be first reported to the state through a lab rather than through the testing provider, so state surveillance staff do not wait to receive the ACRF from the medical provider, which may be delayed or never arrive. Rather the data regarding the individual receiving a positive test are entered into the state surveillance system after verifying it via an initial call to the provider by a state surveillance coordinator or via interview with the PLWH.

Interview participants reported that in addition to the information collected for HIV surveillance and put into eHARS, their states often use other data sources that include information about gender identity to complete these fields, 
including web-based systems used for STD surveillance, partner tracking, and partner notification such as Patient Reporting Investigation Surveillance Manager (PRISM; STD surveillance and contact tracking data system) and Maven Disease Surveillance and Outbreak Management System (provides a system of STD reporting and data management) (Conduent, n.d.; Shiver et al., 2009). In addition, states use lab reports from companies such as Lab Corp and physician notes to track gender identity. Finally, states were reported to use systems for collecting data on individuals living with HIV including CAREWare, the system for collecting data for the Ryan White Care Act program, to supplement missing gender identity data (HRSA, 2019).

Interview participants described another method of obtaining gender identity information that involves medical practices allowing surveillance staff to access their electronic medical records (EMR) to search for gender identity notes. However, since EMR do not always contain the necessary gender identity fields or may have incomplete information, record abstractors must sometimes look further through provider notes for any information about gender identity. It was reported that providers often do not collect gender identity information in any uniform way.

Finally, interview participants reported that information regarding gender identity is frequently captured or verified through DIS in interviews with individuals newly diagnosed with HIV or by care coordinators locating individuals living with HIV who have discontinued medical care. In some states, this information is systematically transferred to the surveillance systems; in others, systemic barriers to data sharing were reported.

\section{Psychosocial and Political Barriers to Identifying Gender Identity Among Individuals Testing Positive for HIV}

State interviewees identified additional barriers to obtaining information about gender identity among gender minorities testing positive for HIV that were related to HIV care accessibility and acceptability. These barriers included hesitance to reveal HIV status and/or gender identity to those collecting data because of fears of confidentiality loss and/or discrimination, and lack of access to HIV primary care and prevention. These barriers were also reported to result in greater reluctance to participate in HIV testing.

It was also frequently reported that some staff involved in testing administration and documentation have an inadequate understanding of social determinants that affect transgender health and that this lack of knowledge may result in less compassion and lowered investment in determining accurate gender identity. Interview participants also reported that some staff, including DIS, may lack important communication skills around transgender issues or are uncomfortable asking questions about a person's gender identity.
An additional reported barrier was that because transgender issues are politicized and deemed controversial by some at the local and state policy/governmental levels, it can be challenging to change a simple HIV-related data collection form or to ask more questions of an individual receiving HIV testing. Furthermore, bureaucratic processes were reported to act as barriers to making changes to forms or systems that could improve gender identity reporting.

Interview participants also discussed how the present day divisive political climates in many states results in greater difficulty for state staff to advocate for more measures inclusive of gender identity for data collection and programming.

\section{Respondent Suggestions for Improving Data Collection Related to Gender Identity}

The consensus of interview participants was that having accurate information on gender identity in HIV surveillance data was critical to planning for HIV prevention and care needs. Improving data collection and cultural sensitivity were identified by respondents as strategies to improve completion and accuracy of this data. Respondents noted that these strategies would require leadership, funding, staff buy-in, and commitment to change from multiple types of entities.

\section{Data Collection}

Notably, all interview respondents indicated a need for changes to improve data collection related to gender identity. Most interview respondents reported that changes to and greater standardization of surveillance forms and data systems used for HIV testing and testing follow-up were needed so that they could more accurately capture the information necessary to determine gender identity. Specific suggestions included:

- Implementing steps to enhance gender identity collection at the time of testing, which would allow for a reduction of inaccurate data and/or inefficient methods of data verification. One suggestion to potentially increase data collection at the time of testing was for the $\mathrm{CDC}$ to require, rather than recommend, completion of gender identity information, optimally using the two-step gender identity method whenever feasible. Assistance with/additional funding for testing and health care provider education regarding capturing gender identity data was also noted as important to increasing gender identity reporting. A respondent stated that, "more education to providers is needed to encourage them to document the transgender information in case notes, and to record the current gender when known on the ACRF."

- Financial and Technical Assistance (TA) support for states to improve data collection systems and to include 
mechanisms for the various data systems that collect information on gender identity to be able to sync with one another was indicated as a significant need. This included assistance with data collection programming. A participant stated that, "SAS (data management analysis software) programs need to be written to incorporate database matching of eHARS and the HIV care databases to identify any undocumented transgender cases in eHARS. Once written, matches for these variables will become routine." A respondent reported that the CDC has assisted with this type of data streamlining in the past, as they released standardized SAS codes for states to help identify who might have discrepant gender and sex assigned at birth information.

- Encouraging or even requiring health care facilities and laboratories to build their electronic medical records to include gender identity questions. One participant said, "the results of the questions could be reviewed by individuals doing chart abstractions and also could be electronically submitted to the health department."

- Addition of a "non-binary" category to the question inquiring about current gender identity on the ACRF was frequently noted as important to depicting diverse gender identity categories more accurately.

\section{Cultural Sensitivity}

Cultural sensitivity trainings for medical staff and social service providers (including testing professionals and DIS) were also suggested as a beneficial strategy to better capture accurate gender identity data. Specific issues and suggestions included:

- $T A$ and funding support to enhance $L G B T Q+$ cultural sensitivity training for individuals involved in testing, DIS, and related data collection. This would include providing routine in-person and web-based training on transphobia, homophobia, and undoing racism for individuals involved in collecting gender identity data.

- Cultural sensitivity training for care providers: Interview participants said that many medical providers, especially at university and HIV specialty clinics seem skilled and sensitive when asking questions about gender identity. However, respondents also reported that there are some providers who do not ask basic questions about gender identity, thus training is needed to increase cultural sensitivity among medical providers. One respondent said, "We need cultural competence with medical providers."

- Focus on rural areas: Interview participants reported that medical care providers/staff in rural areas are often less knowledgeable about gender identity. It was reported that, in some cases, providers had culturally inappropriate responses to transgender patients and created mistrust that lingered for these individuals. Interview participants suggested trainings for smaller, rural clinics on gender identity to improve cultural sensitivity. One respondent noted that this information needs to be reinforced repeatedly, saying, "It will take more than one training to change attitudes about transgender issues."

- Community involvement: Several respondents suggested that ongoing engagement of transgender and non-binary people in the development and guidance of surveillance systems, and data collection forms will support increased completion of gender identity data fields. They stated that this guidance is also essential in developing cultural sensitivity programming that both educates and assists providers in creating environments that are safe for transgender individuals to disclose their gender identity and receive the care they need.

\section{Conclusions}

The US Healthy People 2030 goals for the LGBT population state the importance of increasing the number of states that include questions that identify sexual and gender identity in state level surveys and data systems in an attempt to address the lack of comprehensive data for this population (Healthy People 2030, n.d.). Other policy research organizations, such as the University of California San Francisco Center of Excellence for Transgender Health and the Council of State and Territorial Epidemiologists, have reported the necessity of having these data available for HIV prevention and care planning (Council of State and Territorial Epidemiologist, 2016; Sausa et al., 2009). Furthermore, a CDC report on HIV among transgender communities stated that accurate gender identity data are necessary for developing and implementing HIV prevention programs, but that there has been limited national information on HIV among transgender and non-binary populations. The CDC authors state, "In large part, this is because there has been no reliable system for collecting and sharing both sex and gender identity information in health records." (CDC, 2019c). Interviews with state epidemiology staff in the Deep South indicate that significant effort is needed to achieve the goal of improved availability of gender identity information in HIV surveillance data in this region, as gender identity information in state HIV surveillance data is currently incomplete and lacks the accuracy needed for service planning. This lack of accurate gender identity data was reportedly due to barriers including lack of standardized data collection strategies and incompatibility among systems for data collection and sharing, lack of sufficient cultural understanding and sensitivity related to gender identity, and lack of consistent collection and/or documentation of the CDC's recommended two-step method for collecting gender identity at the time of 
testing or at the point of medical care. These barriers were described as spanning community testing sites, communitybased organizations, and healthcare entities.

Interview participants reported that identifying ways to effectively prepare and motivate organizations involved in HIV care to collect gender identity data is a critical component of comprehensive gender identity data collection among individuals testing positive for HIV. However, previous studies have found that many healthcare organizations perceived barriers to incorporating a systematic data collection approach for gender identity and sexual orientation. Perceived barriers included making staff and patients uncomfortable, the inability to record the data in their EMR platform, and inadequate dissemination of best practices (Grasso et al., 2019). Researchers found that $77.9 \%$ of providers felt patients would be offended by or refuse questions about sexual orientation and gender identity; however, only $10.3 \%$ of patients actually reported this concern (Haider et al., 2017). Instituting culturally sound systems of data collection and having culturally competent service providers at all levels of care can assist in maximizing individuals' comfort in sharing gender identity information. However, self-determination is critical; thus, systems that appear coercive in acquiring this information must be avoided so that individuals perceive that they maintain the right to withhold this information if they prefer. Because of this selfdetermination requirement, the data can never be fully complete, similar to collection of any type of sensitive data.

Interview participants offered specific recommendations for improving the accuracy and availability of gender identity information in HIV surveillance reporting in the Deep South and other areas in the US with limited gender identity data collection. These recommendations focused on strategies to improve data collection systems and database functionality, such as mechanisms for relevant databases to sync with one another, and educational strategies to improve cultural sensitivity related to gender identity and to enhance motivation and skills to improve accurate collection of this information at time of testing and/or in medical care. For example, to increase cultural capacity, the participants recommended that federal and state funding through CDC and/ or other federal and state agencies, including the Ryan White program, be increased to support evidence-based gender identity cultural sensitivity training for health department staff and medical and social service providers. They also emphasized the need to ensure that programming is suited for and available to rural areas and that it is developed with meaningful involvement of members of the transgender and non-binary communities. There are some examples of existing programs that could be more widely disseminated such as the CDC training on patient-centered health care for transgender individuals (Centers for Disease Control and Prevention, 2019d). The CDC also provides the opportunity to request TA on HIV prevention-related issues through the capacity building branch, which could potentially offer these types of cultural sensitivity trainings (Center for Disease Control and Prevention, 2020b).

It was also recommended that the $\mathrm{CDC}$ and other federal agencies develop formal guidance and provide TA that targets and educates laboratories and other medical organizations on best practices to develop and implement systems of data collection that routinely capture gender identity data while also guaranteeing that respondent confidentiality is protected, and non-discrimination provisions are in place.

Implementation of the recommended strategies from individuals directly working in HIV surveillance systems would offer the opportunity to enhance the availability of gender identity data, which is critical to planning HIV prevention and care strategies in the highly affected Deep South region. The need for appropriately targeting HIV funding and programming based on accurate data has only intensified with the onset of the COVID-19 pandemic, which has deepened health inequalities and added strain to public health budgets in the Deep South.

Findings related to the interviews with HIV surveillance leaders in the Deep South states must be taken in context of their limitations, which include changes in data collection that may have occurred since the interviews and that interviews did not include the perspectives of staff working directly in data collection, including testing providers and DIS workers, who may have different experiences and recommendations than surveillance leadership staff. Acquiring these frontline perspectives will be important to more fully understand the context and to make recommendations for maximizing gender identity data collection. Additional focus and research on gender identity documentation in HIV surveillance will continue to be critical to maximizing the accuracy and completeness of this data and subsequently improving prevention and care for gender minorities.

Funding This report was supported by the Ford Foundation.

\section{Declarations}

Conflict of Interest The authors declare no competing interests.

\section{References}

Alabama Division of STD Prevention and Control HIV Surveillance Branch. (2020). 2019 State of Alabama HIV surveillance annual report. Retrieved from https://www.alabamapublichealth.gov/hiv/ assets/hivsurveillanceannualreport_2019.pdf

Association APHA. (2020). Structural racism is a public health crisis: Impact on the black community. Retrieved from https://www.apha. 
org/policies-and-advocacy/public-health-policy-statements/policydatabase/2021/01/13/structural-racism-is-a-public-health-crisis

Balagi, A. (2019). Email Correspondence. CDC.

Becasen, J., Denard, C., Mullins, M., Higa, D., \& Sipe, T. (2018). Estimating the prevalence of HIV and sexual behaviors among the US transgender population: A systematic review and metaanalysis, 2006-2017. American Journal of Public Health, e1-8. https://doi.org/10.2105/AJPH.2018.304727

Centers for Disease Control and Prevention. (2009). Enhanced HIV/ AIDS reporting system. Retrieved from https://www.cdc.gov/hiv/ library/reports/hiv-surveillance.html

Centers for Disease Control and Prevention. (2018). Adult HIV confidential case report. Retrieved from https://www.cdc.gov/ hiv/pdf/guidelines/cdc-hiv-adult-confidential-case-report-form2018.pdf

Centers for Disease Control and Prevention. (2019a). HIV in the southern United States. Retrieved from https://www.cdc.gov/ hiv/pdf/policies/cdc-hiv-in-the-south-issue-brief.pdf

Center for Disease Control and Prevention. (2019b). NCHHSTP Atlas. Retrieved from http://www.cdc.gov/nchhstp/atlas/. Retrieved April 2019 http://www.cdc.gov/nchhstp/atlas/

Centers for Disease Control ad Prevention. (2019c). HIV and transgender people. Retrieved from Atlanta: https://www.cdc. gov/hiv/group/gender/transgender/index.html

Centers for Disease Control and Prevention. (2019d). Patientcentered care for transgender people: Recommended practices for health care settings. Retrieved from https://www.cdc. gov/hiv/clinicians/transforming-health/health-care-providers/ affirmative-care.html

Centers for Disease Control and Prevention. (2020a). Monitoring selected national HIV prevention and care objectives by using HIV surveillance data-United States and 6 dependent areas, 2018. HIV Surveillance Supplemental Report, 25(No. 2). http:// www.cdc.gov/hiv/library/reports/hiv-surveillance.html. Published May 2020. Accessed January 2022.

Center for Disease Control and Prevention. (2020b). Capacity building branch (CBB). Retrieved from https://www.cdc.gov/hiv/ programresources/capacitybuilding/index.html

Center for Disease Control and Prevention. (2021a). HIV infection, risk, prevention, and testing behaviors among transgender women-National HIV behavioral surveillance, 7 US cities, 2019-2020. Retrieved from https://www.cdc.gov/hiv/pdf/ library/reports/surveillance/cdc-hiv-surveillance-special-reportnumber-27.pdf

Center for Disease Control and Prevention. (2021b). HIV surveillance report. Retrieved from https://www.cdc.gov/hiv/statistics/ overview/index.html\#: : text $=$ At $\% 20$ the $\% 20$ end $\% 20$ of $\%$ 202019 ,infections $\% 20$ had $\% 20$ not $\% 20$ been $\% 20$ diagnosed.\& text $=$ In $\% 2050 \% 20$ states $\% 20$ and $\% 20$ the $\% 20$ District $\% 20$ of $\%$ 20Columbia.\&text=CDC.,the $\% 20$ United $\% 20$ States $\% 202015 \%$ E2\% $80 \% 932019$

Clark, H., Babu, A., Wiewel, E., Opoku, J., \& Crepaz, N. (2017). Diagnosed HIV infection in transgender adults and adolescents: Results from the national HIV Surveillance System. AIDS and Behavior, 21(9), 2774-2783.

Cohen, S., Gray, K., Ocfemia, M., Johnson, A., \& Hall, H. (2014). The status of the national HIV surveillance system, United States, 2013. Public Health Reports, 129(4), 335-341. Retrieved from https://www.ncbi.nlm.nih.gov/pmc/articles/ PMC4037459/\#B11

Conduent. (n.d.). Maven Disease Surveillance and Outbreak Management System. Retrieved from https://downloads.conduent. com/content/usa/en/brochure/maven-outbreak-management.pdf

Council of State and Territorial Epidemiologist. (2016). Transgender HIV surveillance retrieved from https://cdn.ymaws.com/www. cste.org/resource/resmgr/2017PS/2017PSFinal/17-ID-06.pdf
Department of Health, S. H. O. (n.d.). Reported cases of HIV 2014 2018. Retrieved from http://www.msdh.state.ms.us/msdhsite/ index.cfm/14,0,150,807,html\#hiv

DHEC, S. C. (2020). South Carolina Epidemiologic Profile of HIV, AIDS, and sexually transmitted infections 2020. Retrieved from https://scdhec.gov/sites/default/files/media/document/SC-HIVEpi-Profile-2020.pdf

Florida Department of Health. (2020). State of epidemic, 2019, State HIV slide sets. Retrieved from http://www.floridahealth.gov/ diseases-and-conditions/aids/surveillance/epi-slide-sets.html

Grasso, C., McDowell, M., Goldhammer, H., \& Keuroghlian, A. (2019). Planning and implementing sexual orientation and gender identity data collection in electronic health records. Journal of the American Medical Informatics Assocation, 26(1), 66-70.

Haider, H., Schneider, E., \& Kodadek, L. (2017). Emergency department query for patient-centered approaches to sexual orientation and gender identity: The EQUALITY Study. JAMA Internal Medicine, 177(6), 819-828.

Health, G. D. O. P. (2019). HIV surveillance summary, Georgia 2019. Retrieved from https://dph.georgia.gov/epidemiology/georgiashivaids-epidemiology-section/georgia-hiv-surveillancedata

Healthy People 2030. (n.d.). LGBT. Retrieved from https://health. gov/healthypeople/objectives-and-data/browse-objectives/lgbt

HIV/STD/Hepatitis Surveillance Unit NC Department of Health and Human Services. (2020). 2019 North Carolina HIV surveillance report. Retrieved from https://epi.dph.ncdhhs.gov/cd/stds/ figures/hiv19rpt_11302020.pdf

HRSA. (2019). CAREWare. Retrieved from https://hab.hrsa.gov/ program-grants-management/careware

National Center for Transgender Equality. (2019). Issues: Housing \& homelessness. Retrieved from https://transequality.org/issues/ housing-homelessness

Pichon, L. C., Williams Powell, T., Williams Stubbs, A., BectonOdum, N., Ogg, S., Arnold, T., \& Thurston, I. B. (2020). An exploration of U.S. Southern Faith Leaders' perspectives of HIV prevention, xexuality, and sexual health teachings. International journal of environmental research and public health, 17(16), 5734. https://doi.org/10.3390/ijerph17165734

Reif, S., Safley, D., Wilson, E., \& Whetten, K. (2016). HIV/AIDS in the U.S. Deep South: Trends from 2008-2013. Retrieved from https://southernaids.files.wordpress.com/2011/10/hiv-aids-inthe-us-deep-south-trends-from-2008-2013.pdf

Reif, S., Whetten, K., Wilson, E., McAllaster, C., Pence, B., LeGrand, S., \& Gong, W. (2014). HIV/AIDS in Southern USA: A disproportionate epidemic. AIDS Care, 26(3), 351-359.

Reif, S., \& Wilson, E. (2017). Interventions to address HIV-related stigma: Literature summary. Retrieved from https://southernaidsstrategy. org/hiv-related-stigma-research/

Sausa, L., Sevelius, J., Keatley, J., Iniquez, J., \& Reyes, M. (2009). Policy recommendations for inclusive data collection of trans people in HIV prevention, care \& services. Retrieved from https://prevention.ucsf.edu/transhealth/education/ data-recs-long

Shiver, S., Schmitt, K., \& Cooksey, A. (2009). Use of a business approach to improve disease surveillance data management systems and information technology process in Florida's Bureau of STD Prevention and Control. Public Health Reports, 124, 98-102. https://doi.org/10.1177/00333549091240S214

State of Louisiana Department of Health. (2020). 2018 STD/HIV surveillance report. Retrieved from https://ldh.la.gov/assets/oph/ HIVSTD/2018_STDHIV_SurveillanceReport_Final.pdf

TB/HIV/STD Epidemiology and Surveillance Branch. (2021). Texas HIV surveillance report, 2019 annual report. Retrieved from https://www.dshs.texas.gov/hivstd/reports/HIVSurveillance Report.pdf 
Tennessee Department of Health. (2021). Tennessee HIV epidemiologic profile, 2019. Retrieved from https://www.tn.gov/ content/dam/tn/health/program-areas/hiv/2019-Tennessee-HIVEpidemiological-Profile.pdf
Publisher's Note Springer Nature remains neutral with regard to jurisdictional claims in published maps and institutional affiliations. 